\title{
On the Stability and Boundedness of Solutions of Certain Non-Autonomous Delay Differential Equation of Third Order
}

\author{
Akinwale L. Olutimo ${ }^{*}$, Daniel 0. Adams ${ }^{2}$ \\ ${ }^{1}$ Department of Mathematics, Lagos State University, Ojo, Nigeria \\ ${ }^{2}$ Department of Mathematics, Federal University of Agriculture, Abeokuta, Nigeria \\ Email: *aolutimo@yahoo.com, danielogic2008@yahoo.com
}

Received 23 January 2016; accepted 21 March 2016; published 24 March 2016

Copyright (C) 2016 by authors and Scientific Research Publishing Inc.

This work is licensed under the Creative Commons Attribution International License (CC BY).

http://creativecommons.org/licenses/by/4.0/

(c) (i) Open Access

\section{Abstract}

In this paper, we study certain non-autonomous third order delay differential equations with continuous deviating argument and established sufficient conditions for the stability and boundedness of solutions of the equations. The conditions stated complement previously known results. Example is also given to illustrate the correctness and significance of the result obtained.

\section{Keywords}

Asymptotic Stability, Boundedness, Lyapunov Functional, Delay Differential Equations, Third-Order Delay Differential Equations

\section{Introduction}

This paper considers the third order non-autonomous nonlinear delay differential

$$
\begin{aligned}
& x^{\prime \prime \prime}(t)+a(t) h\left(x(t), x^{\prime}(t)\right) x^{\prime \prime}(t)+b(t) g\left(x^{\prime}(t-r(t))\right)+c(t) f(x(t-r(t))) \\
& =p\left(t, x(t), x^{\prime}(t), x(t-r(t)), x^{\prime}(t-r(t)), x^{\prime \prime}(t)\right)
\end{aligned}
$$

or its equivalent system

"Corresponding author.

How to cite this paper: Olutimo, A.L. and Adams, D.O. (2016) On the Stability and Boundedness of Solutions of Certain Non-Autonomous Delay Differential Equation of Third Order. Applied Mathematics, 7, 457-467. 


$$
\begin{aligned}
x^{\prime}= & y, \\
y^{\prime}= & z, \\
z^{\prime}= & -a(t) h(x, y) z-b(t) g(y)-c(t) f(x)+b(t) \int_{t-r(t)}^{t} g^{\prime}(y(s)) z(s) \mathrm{d} s \\
& +c(t) \int_{t-r(t)}^{t} f^{\prime}(x(s)) y(s) \mathrm{d} s+p(t, x, y, x(t-r(t)), y(t-r(t)), z),
\end{aligned}
$$

where $0 \leq r(t) \leq \gamma, r^{\prime}(t) \leq \beta, 0<\beta<1, \beta$ and $\gamma$ are some positive constants, $\gamma$ will be determined later, $a(t), b(t), c(t), h(x, y), g(y), f(x), p(t, x, y, x(t-r(t)), y(t-r(t)), z), t \in \mathbb{R}^{+}, \mathbb{R}^{+}=[0, \infty)$ are real valued functions continuous in their respective arguments on $\mathbb{R}^{+}, \mathbb{R}^{+}, \mathbb{R}^{+}, \mathbb{R}^{2}, \mathbb{R}, \mathbb{R}$ and $\mathbb{R}^{+} \times \mathbb{R}^{5}$ respectively and $\mathbb{R}^{+}=[0, \infty)$. Besides, it is supposed that the derivatives $f^{\prime}(x), g^{\prime}(y)$ are continuous for all $x, y$, with $f(0)=g(0)=0$. In addition, it is also assumed that the functions $h(x, y), f(x(t-r(t)))$, $g\left(x^{\prime}(t-r(t))\right)$ and $p(t, x, y, x(t-r(t)), y(t-r(t)), z)$ satisfy a Lipschitz condition in $x, y, x(t-r(t))$, $y(t-r(t))$ and $z$; throughout the paper $x(t), y(t)$ and $z(t)$ are respectively abbreviated as $x, y$ and $z$. Then the solutions of (1.1) are unique.

In applied science, some practical problems are associated with Equation (1.1) such as after effect, nonlinear oscillations, biological systems and equations with deviating arguments (see [1]-[3]). It is well known that the stability of solutions plays a key role in characterizing the behavior of nonlinear delay differential equations. Stability is much more complicated for delay equations. Thus, it is worthwhile to continue to investigate the stability and boundedness of solutions of Equation (1.1) and its various forms.

Equation of the form (1.1) in which $a(t), b(t)$ and $c(t)$ are constants has been studied by several authors Sadek [4] [5], Zhu [6], Afuwape and Omeike [7], Ademola and Aramowo [8], Yao and Meng [9], Tunc [3] and Ademola et al [10] to mention a few. They obtain the stability, uniform boundedness and uniform ultimate boundedness of solutions. In a sequence of results, Omeike [11] considers the following nonlinear delay differential equation of the third order, with a constant deviating argument $r$,

$$
x^{\prime \prime \prime}(t)+a(t) x^{\prime \prime}(t)+b(t) g\left(x^{\prime}(t)\right)+c(t) h(x(t-r))=p(t)
$$

and established conditions for the stability and boundedness of solution when $p(t)=0$ and $p(t) \neq 0$ while Tunc [12] considers a similar system with a constant deviating argument $r$ of the form

$$
\begin{aligned}
& x^{\prime \prime \prime}(t)+a(t) \psi(x(t)) x^{\prime \prime}(t)+b(t) g\left(x^{\prime}(t)\right)+c(t) h(x(t-r)) \\
& =p\left(t, x(t), x(t-r), x^{\prime}(t), x^{\prime}(t-r), x^{\prime \prime}(t)\right)
\end{aligned}
$$

and obtains the conditions for its boundedness of solution.

Results obtained are now extended to non-autonomous delay differential Equation (1.1). Results obtained in this work are comparable in generality to the results of Sadek [7] on analogous third order differential equation which itself generalizes an analogous third-order results of Zhu [5], and also complement existing results on third order delay differential equations. We establish sufficient conditions for the stability (when $p \equiv 0$ ) and boundedness (when $p \neq 0$ ) of solutions of Equation (1.1) which extend and improve the results of Omeike [11] and Tunc [12]. An example is given to illustrate the correctness and significance of the result obtained.

Now, we will state the stability criteria for the general non-autonomous delay differential system. We consider:

$$
\dot{x}=f(t, x), x_{t}=x(t+\theta) \quad-r \leq \theta \leq 0, t \geq 0,
$$

where $f: \mathbf{I} \times C_{H} \rightarrow \mathbb{R}^{n}$ is a continuous mapping,

$$
f(t, 0)=0, C_{H}:=\left\{\phi \in\left(C[-r, 0], \mathbb{R}^{n}\right):\|\phi\| \leq H\right\}
$$

and for $H_{1} \leq H$, there exists $L\left(H_{1}\right)>0$, with

$$
|f(\phi)| \leq L\left(H_{1}\right) \text { when }\|\phi\| \leq H_{1} .
$$

Definition 1.0.1 ([8]) An element $\psi \in C$ is in the $\omega$-limit set of $\phi$, say, $\Omega(\phi)$, if $x(t, 0, \phi)$ is defined on $[0, \infty)$ and there is a sequence $\left\{t_{n}\right\}, t_{n} \rightarrow \infty$ as $n \rightarrow \infty$, with $\left\|x_{t_{n}}(\phi)-\psi\right\| \rightarrow 0$ as $n \rightarrow \infty$ where 


$$
x_{t n}(\phi)=x\left(t_{n}+\theta, 0, \phi\right) \text { for }-r \leq \theta \leq 0 .
$$

Definition 1.0.2 ([8] [13]) A set $Q \in C_{H}$ is an invariant set if for any $\phi \in Q$, the solution of (1.2), $x(t, 0, \phi)$, is defined on $[0, \infty)$ and $x_{t}(\phi) \in Q$ for $t \in[0, \infty)$.

Lemma 1 ([8,13]) An element $\phi \in C_{H}$ is such that the solution $x_{t}(\phi)$ of $(1.3)$ with $x_{o}(\phi)=\phi$ is defined on $[0, \infty)$ and $\left\|x_{t}(\phi)\right\| \leq H_{1}<H$ for $t \in[0, \infty)$, then $\Omega(\phi)$ is a non-empty, compact, invariant set and

$$
\operatorname{dist}\left(x_{t}(\phi), \Omega(\phi)\right) \rightarrow 0 \text { as } t \rightarrow \infty \text {. }
$$

Lemma 2 ([8] [13]) Let $V(t, \phi): I \times C_{H} \rightarrow \mathbb{R}$ be a continuous functional satisfying a local Lipschitz condition. $V(t, \phi)=0$, and such that:

1) $W_{1}|\phi(0)| \leq V(t, \phi) \leq W_{2}\|\phi\|$ where $W_{1}(r), W_{2}(r)$ are wedges;

2) $\dot{V}_{(1.3)}(t, \phi) \leq 0$ for $\phi \leq C_{H}$.

Then the zero solution of (1.3) is uniformly stable. If we define $Z=\left\{\phi \in C_{H}: V_{(1.3)}(t, \phi)=0\right\}$, then the zero solution of (1.3) is asymptotically stable provided that the largest invariant set in $Z$ is $Q=\{0\}$.

The following will be our main stability result (when $p \equiv 0$ ) for (1.1).

\section{Statement of Results}

Theorem 1 In addition to the basic assumptions imposed on the functions $a(t), b(t), c(t), h(x, y), g(y), f(x)$ and $p$, let us assume that there exist positive constants $\delta_{1}, \ell_{1}, \ell_{2}, a_{o}, a, b, c, \mu, d_{o}, M_{1}$ and $M_{2}$ such that the following conditions are satisfied:

1) $f^{\prime}(x) \leq c ; c>0, f(0)=0, \frac{f(x)}{x} \geq \delta_{1}>0, \quad x \neq 0 ;$

2) $h(x, y) \geq 1+a ; g(0)=0, \frac{g(y)}{y} \geq b>0, \quad y \neq 0$;

3) $0<\ell_{1} \leq c(t) \leq b(t) \leq d_{o} ;-d_{o} \leq b^{\prime}(t) \leq c^{\prime}(t) \leq 0$ and $0<a_{o} \leq a(t) \leq d_{o}$;

4) $y h_{x}(x, y) \leq 0 ; \frac{1}{2} a^{\prime}(t) \leq \ell_{2}<\ell_{1}(b-\mu c)$ and $\left|f^{\prime}(x)\right| \leq M_{1},\left|g^{\prime}(y)\right| \leq M_{2}$, for all $x, y$.

Then, the zero solution of system (1.2) is asymptotically stable, provided that

$$
\frac{b}{c}>\mu>\frac{1}{a_{o}}
$$

and

$$
\gamma<\min \left\{\frac{\left|2\left[\ell_{2}-\ell_{1}(b-\mu c)\right](1-\beta)\right|}{d_{o}\left(M_{1}+M_{2}\right)(1-\beta)+d_{o} M_{1}(\mu+1)} ; \frac{2\left[\mu a_{o}(1+a)-1\right](1-\beta)}{\mu d_{o}\left(M_{1}+M_{2}\right)(1-\beta)+d_{o} M_{2}(\mu+1)}\right\} .
$$

\section{Proof}

Our main tool is the following Lyapunov functional $V=V\left(x_{t}, y_{t}, z_{t}\right)$ defined as

$$
\begin{aligned}
2 V\left(x_{t}, y_{t}, z_{t}\right)= & 2 c(t) \int_{0}^{x} f(\xi) \mathrm{d} \xi+2 \mu b(t) \int_{0}^{y} g(\eta) \mathrm{d} \eta \\
& +2 a(t) \int_{0}^{y}[h(x, \eta)-a] \eta \mathrm{d} \eta+\mu z^{2}+2 y z+2 \mu c(t) f(x) y \\
& +2 \lambda \int_{-r(t)}^{0} \int_{t+s}^{t} y^{2}(\theta) \mathrm{d} \theta \mathrm{d} s+2 \delta \int_{-r(t)}^{0} \int_{t+s}^{t} z^{2}(\theta) \mathrm{d} \theta \mathrm{d} s,
\end{aligned}
$$

where $\lambda$ and $\delta$ are positive constants which will be determined later.

We also assume that

$$
\lim _{t \rightarrow \infty} c(t)=c_{o}, \quad \lim _{t \rightarrow \infty} b(t)=b_{o},
$$

where $0<\ell_{1} \leq c_{o} \leq b_{o} \leq d_{o}$. 
By the assumption $a(t)>0$ and $h(x, y) \geq 1+a$, from (2.3) we have

$$
\begin{aligned}
2 V\left(x_{t}, y_{t}, z_{t}\right) \geq & 2 c(t) \int_{0}^{x} f(\xi) \mathrm{d} \xi+2 \mu b(t) \int_{0}^{y} g(\eta) \mathrm{d} \eta \\
& +a(t) y^{2}+\mu z^{2}+2 y z+2 \mu c(t) f(x) y \\
& +2 \lambda \int_{-r(t)}^{0} \int_{t+s}^{t} y^{2}(\theta) \mathrm{d} \theta \mathrm{d} s+2 \delta \int_{-r(t)}^{0} \int_{t+s}^{t} z^{2}(\theta) \mathrm{d} \theta \mathrm{d} s .
\end{aligned}
$$

The Lyapunov functional (2.4) can be arranged in the form

$$
\begin{aligned}
V \geq & \left(a^{\frac{1}{2}}(t) y+\frac{z}{2 a^{\frac{1}{2}}(t)}\right)^{2}+\frac{1}{4 a(t)}(2 \mu a(t)-1) z^{2} \\
& +\mu b(t) c(t) \int_{0}^{y}\left\{\frac{1}{c(t)}\left[\frac{g(\eta)}{\eta}\right]-\frac{b}{b(t)}\right\} \eta \mathrm{d} \eta \\
& +\mu \frac{c(t)}{b}\{f(x)+b y\}^{2}+c(t) \int_{0}^{x}\left[1-\frac{\mu}{b} f^{\prime}(\xi)\right] f(\xi) \mathrm{d} \xi \\
& +\lambda \int_{-r(t)}^{0} \int_{t+s}^{t} y^{2}(\theta) \mathrm{d} \theta \mathrm{d} s+\delta \int_{-r(t)}^{0} \int_{t+s}^{t} z^{2}(\theta) \mathrm{d} \theta \mathrm{d} s .
\end{aligned}
$$

From Theorem 1, $\mu a(t) \geq \mu a_{o}>1$ and $\mu>\frac{1}{a_{o}}$ which makes $2 \mu a(t)-1>0$.

Thus, there is a $\delta_{2}>0$ such that

$$
\left(a^{\frac{1}{2}}(t) y+\frac{z}{2 a^{\frac{1}{2}}(t)}\right)^{2}+\frac{1}{4 a(t)}(2 \mu a(t)-1) z^{2} \geq \frac{1}{2} \delta_{2} y^{2}+\frac{1}{2} \delta_{2} z^{2} .
$$

By (2) and (3) of Theorem 1, we have that the third term on the right in (2.5)

$$
\mu b(t) c(t) \int_{0}^{y}\left\{\frac{1}{c(t)}\left[\frac{g(\eta)}{\eta}\right]-\frac{b}{b(t)}\right\} \eta \mathrm{d} \eta \geq 0,
$$

and next two terms give

$$
\begin{aligned}
& \mu \frac{c(t)}{b}\{f(x)+b y\}^{2}+c(t) \int_{0}^{x}\left[1-\frac{\mu}{b} f^{\prime}(\xi)\right] f(\xi) \mathrm{d} \xi \\
& \geq c(t) \int_{0}^{x}\left[1-\frac{\mu}{b} f^{\prime}(\xi)\right] f(\xi) \mathrm{d} \xi .
\end{aligned}
$$

Using (2.6), (2.7) and (2.8) in (2.5), we have

$$
\begin{aligned}
V\left(x_{t}, y_{t}, z_{t}\right) \geq & c(t) \int_{0}^{x}\left[1-\frac{\mu}{b} f^{\prime}(\xi)\right] f(\xi) \mathrm{d} \xi+\frac{1}{2} \delta_{2} y^{2}+\frac{1}{2} \delta_{2} z^{2} \\
& +\lambda \int_{-r(t)}^{0} \int_{t+s}^{t} y^{2}(\theta) \mathrm{d} \theta \mathrm{d} s+\delta \int_{-r(t)}^{0} \int_{t+s}^{t} z^{2}(\theta) \mathrm{d} \theta \mathrm{d} s . \\
\geq & d_{1} x^{2}+\frac{1}{2} \delta_{2} y^{2}+\frac{1}{2} \delta_{2} z^{2}+\lambda \int_{-r(t)}^{0} \int_{t+s}^{t} y^{2}(\theta) \mathrm{d} \theta \mathrm{d} s+\delta \int_{-r(t)}^{0} \int_{t+s}^{t} z^{2}(\theta) \mathrm{d} \theta \mathrm{d} s,
\end{aligned}
$$

where

$$
d_{1}=\frac{\delta_{1} \ell_{1} \delta_{3}}{2}
$$


and integrals

$$
\lambda \int_{-r(t)}^{0} \int_{t+s}^{t} y^{2}(\theta) \mathrm{d} \theta \mathrm{d} s \text { and } \delta \int_{-r(t)}^{0} \int_{t+s}^{t} z^{2}(\theta) \mathrm{d} \theta \mathrm{d} s \text { are non-negative. }
$$

Thus, for some positive constants $\delta_{1} \ell_{1} \delta_{3}$ and $\delta_{2}$, where $D_{1}=\left\{\frac{1}{2}\left(\delta_{1} \ell_{1} \delta_{3}\right), \frac{1}{2} \delta_{2}, \frac{1}{2} \delta_{2}\right\}$ small enough such that

$$
V\left(x_{t}, y_{t}, z_{t}\right) \geq D_{1}\left(x^{2}+y^{2}+z^{2}\right) .
$$

For the time derivative of the Lyapunov functional (2.3), along a trajectory of the system (1.2), we have

$$
\begin{aligned}
\frac{\mathrm{d}}{\mathrm{d} t} V\left(x_{t}, y_{t}, z_{t}\right)= & c^{\prime}(t) \int_{0}^{x} f(\xi) \mathrm{d} \xi+\mu b^{\prime}(t) \int_{0}^{x} g(\eta) \mathrm{d} \eta+\mu c^{\prime}(t) f(x) y+\frac{1}{2} a^{\prime}(t) \int_{0}^{y}[h(x, \eta)-a] \eta \mathrm{d} \eta \\
& -\left[b(t) g(y) y-\mu c(t) f^{\prime}(x) y^{2}\right]-[\mu a(t) h(x, y)-1] z^{2}+a(t) y \int_{0}^{y} h_{x}(x, \eta) \eta \mathrm{d} \eta \\
& +b(t) y \int_{t-r(t)}^{t} g^{\prime}(y(s)) z(s) \mathrm{d} s+c(t) y \int_{t-r(t)}^{t} f^{\prime}(x(s)) y(s) \mathrm{d} s \\
& +\mu b(t) z \int_{t-r(t)}^{t} g^{\prime}(y(s)) z(s) \mathrm{d} s+\mu c(t) z \int_{t-r(t)}^{t} f^{\prime}(x(s)) y(s) \mathrm{d} s \\
& +\lambda r(t) y^{2}+\delta r(t) z^{2}+\lambda r^{\prime}(t) \int_{t-r(t)}^{t} y^{2}(s) \mathrm{d} s+\delta r^{\prime}(t) \int_{t-r(t)}^{t} z^{2}(s) \mathrm{d} s \\
& -\lambda \int_{t-r(t)}^{t} y^{2}(s) \mathrm{d} s-\delta \int_{t-r(t)}^{t} z^{2}(s) \mathrm{d} s .
\end{aligned}
$$

From (4) of Theorem 1, $\left|f^{\prime}(x)\right| \leq M_{1},\left|g^{\prime}(y)\right| \leq M_{2}$ and using $2 u v \leq u^{2}+v^{2}$, we have that

$$
\begin{aligned}
& c(t) y \int_{t-r(t)}^{t} f^{\prime}(x(s)) y(s) \mathrm{d} s \leq d_{o} M_{1}|y| \int_{t-r(t)}^{t}|y(s)| \mathrm{d} s \\
& \leq \frac{1}{2} d_{o} M_{1} r(t) y^{2}+\frac{1}{2} d_{o} M_{1} \int_{t-r(t)}^{t} y^{2}(s) \mathrm{d} s . \\
& \mu c(t) z \int_{t-r(t)}^{t} f^{\prime}(x(s)) y(s) \mathrm{d} s \leq \mu d_{o} M_{1}|z| \int_{t-r(t)}^{t}|y(s)| \mathrm{d} s \\
& \leq \frac{1}{2} \mu d_{o} M_{1} r(t) z^{2}+\frac{1}{2} \mu d_{o} M_{1} \int_{t-r(t)}^{t} y^{2}(s) \mathrm{d} s
\end{aligned}
$$

Similarly, we obtain

$$
\begin{aligned}
& b(t) y \int_{t-r(t)}^{t} g^{\prime}(y(s)) z(s) \mathrm{d} s \leq \frac{1}{2} d_{o} M_{2} r(t) y^{2}+\frac{1}{2} d_{o} M_{2} \int_{t-r(t)}^{t} z^{2}(s) \mathrm{d} s \\
& \mu b(t) z \int_{t-r(t)}^{t} g^{\prime}(y(s)) z(s) \mathrm{d} s \leq \frac{1}{2} \mu d_{o} M_{2} r(t) z^{2}+\frac{1}{2} \mu d_{o} M_{2} \int_{t-r(t)}^{t} z^{2}(s) \mathrm{d} s
\end{aligned}
$$

Thus,

$$
\begin{aligned}
\frac{\mathrm{d}}{\mathrm{d} t} V\left(x_{t}, y_{t}, z_{t}\right) \leq & c^{\prime}(t) \int_{0}^{x} f(\xi) \mathrm{d} \xi+\mu b^{\prime}(t) \int_{0}^{x} g(\eta) \mathrm{d} \eta+\mu c^{\prime}(t) f(x) y+\frac{1}{2} a^{\prime}(t) \int_{0}^{y}[h(x, \eta)-a] \eta \mathrm{d} \eta \\
& -\left[b(t) g(y) y-\mu c(t) f^{\prime}(x) y^{2}\right]-[\mu a(t) h(x, y)-1] z^{2}+a(t) y \int_{0}^{y} h_{x}(x, \eta) \eta \mathrm{d} \eta \\
& +\frac{1}{2}\left(d_{o} M_{2}+d_{o} M_{1}+\lambda\right) r(t) y^{2}+\frac{1}{2}\left(\mu d_{o} M_{2}+\mu d_{o} M_{1}+\delta\right) r(t) z^{2} \\
& +\left[\frac{1}{2} d_{o} M_{1}(\mu+1)-\lambda\left(1-r^{\prime}(t)\right)\right] \times \int_{t-r(t)}^{t} y^{2}(s) \mathrm{d} s \\
& +\left[\frac{1}{2} d_{o} M_{2}(\mu+1)-\delta\left(1-r^{\prime}(t)\right)\right] \times \int_{t-r(t)}^{t} z^{2}(s) \mathrm{d} s .
\end{aligned}
$$


If $y=0$, then $b(t) g(y) y-\mu c(t) f^{\prime}(x) y^{2}=0$. If $y \neq 0$, we can rewrite the term as

$$
\left[b(t) \frac{g(y)}{y}-\mu c(t) f^{\prime}(x)\right] y^{2} \geq[b b(t)-\mu c c(t)] y^{2} \geq \ell_{1}[b-\mu c] y^{2},
$$

where by (3) of Theorem $1, \quad b(t) \geq c(t) \geq \ell_{1}>0$.

And by (1) and (2) of Theorem 1,

$$
\frac{1}{2} a^{\prime}(t) \int_{0}^{y}[h(x, \eta)-a] \eta \mathrm{d} \eta-\left[b(t) \frac{g(y)}{y}-\mu c(t) f^{\prime}(x)\right] y^{2}
$$

as

$$
\begin{aligned}
& \leq \frac{1}{2} a^{\prime}(t) y^{2}-\left[b(t) \frac{g(y)}{y}-\mu c(t) f^{\prime}(x)\right] y^{2} \\
& \leq\left[\ell_{2}-\ell_{1}(b-\mu c)\right] y^{2} .
\end{aligned}
$$

According to (2) of Theorem 1, $h(x, y) \geq 1+a$ and by (3), $\mu a(t) \geq \mu a_{o}>1$ and certainly $\mu a_{o}(1+a) \gg 1$ thus,

$$
\begin{aligned}
& {[\mu a(t) h(x, y)-1] z^{2} \leq\left(\mu a_{o}(1+a)-1\right) z^{2},} \\
& a(t) y \int_{0}^{y} h_{x}(x, \eta) \eta \mathrm{d} \eta \leq 0
\end{aligned}
$$

and by (3) and (4) of Theorem 1, we have that

$$
c^{\prime}(t) \int_{0}^{x} f(\xi) \mathrm{d} \xi+\mu b^{\prime}(t) \int_{0}^{x} g(\eta) \mathrm{d} \eta+\mu c^{\prime}(t) f(x) y \leq 0
$$

for all $x, y$ and $t \geq 0$.

Thus, from (2.11), (2.12), (2.13), (2.14) and (2.15), we have

$$
\begin{aligned}
\frac{\mathrm{d}}{\mathrm{d} t} V\left(x_{t}, y_{t}, z_{t}\right) \leq & -\left[\ell_{2}-\ell_{1}(b-\mu c)\right] y^{2}-\left[\mu a_{o}(1+a)-1\right] z^{2} \\
& +\frac{1}{2}\left(d_{o} M_{2}+d_{o} M_{1}+\lambda\right) r(t) y^{2}+\frac{1}{2}\left(\mu d_{o} M_{2}+\mu d_{o} M_{1}+\delta\right) r(t) z^{2} \\
& +\left[\frac{1}{2} d_{o} M_{1}(\mu+1)-\lambda(1-\beta)\right] \times \int_{t-r(t)}^{t} y^{2}(s) \mathrm{d} s \\
& +\left[\frac{1}{2} d_{o} M_{2}(\mu+1)-\delta(1-\beta)\right] \times \int_{t-r(t)}^{t} z^{2}(s) \mathrm{d} s .
\end{aligned}
$$

If we choose

$$
\lambda=\frac{d_{o} M_{1}(\mu+1)}{2(1-\beta)}>0
$$

and

$$
\delta=\frac{d_{o} M_{2}(\mu+1)}{2(1-\beta)}>0
$$

and using $r(t)$, we obtain

$$
\begin{aligned}
\frac{\mathrm{d}}{\mathrm{d} t} V\left(x_{t}, y_{t}, z_{t}\right) \leq & -\frac{1}{2}\left\{2\left[\ell_{2}-\ell_{1}(b-\mu c)\right]-\frac{\gamma\left[d_{o}\left(M_{2}+M_{1}\right)(1-\beta)+d_{o} M_{1}(\mu+1)\right]}{(1-\beta)}\right\} y^{2} \\
& -\frac{1}{2}\left\{2\left[\mu a_{o}(1+a)-1\right]-\frac{\gamma\left[\mu d_{o}\left(M_{2}+M_{1}\right)(1-\beta)+d_{o} M_{2}(\mu+1)\right]}{(1-\beta)}\right\} z^{2} .
\end{aligned}
$$


Choosing

$$
\gamma<\min \left\{\frac{\left|2\left[\ell_{2}-\ell_{1}(b-\mu c)\right](1-\beta)\right|}{d_{o}\left(M_{2}+M_{1}\right)(1-\beta)+d_{o} M_{1}(\mu+1)} ; \frac{2\left[\mu a_{o}(1+a)-1\right](1-\beta)}{\mu d_{o}\left(M_{2}+M_{1}\right)(1-\beta)+d_{o} M_{2}(\mu+1)}\right\},
$$

we have

$$
\frac{\mathrm{d}}{\mathrm{d} t} V\left(x_{t}, y_{t}, z_{t}\right) \leq-\delta_{4}\left(y^{2}+z^{2}\right),
$$

for some $\delta_{4}>0$.

Finally, it follows that $\frac{\mathrm{d}}{\mathrm{d} t} V\left(x_{t}, y_{t}, z_{t}\right)=0$ if and only if $y_{t}=z_{t}=0, \frac{\mathrm{d}}{\mathrm{d} t} V(\phi)<0$ for $\phi \neq 0$ and for $V \geq U(|\phi(0)|) \geq 0$.

Thus, (2.10) and (2.16) and the last statement agreed with Lemma 2. This shows that the trivial solution of (1.1) is asymptotically stable.

Hence, the proof of the Theorem 1 is now complete.

Remark 2.1 If $r(t)$ is a constant and (1.1) is the constant co-efficient delay differential equation $x^{\prime \prime \prime}+a x^{\prime \prime}+b x^{\prime}(t-r)+c x(t-r)=0$, then conditions (1)-(4) reduce to the Routh-Hurwitz conditions $a>0, c>0$ and $a b>c$. To show this we set $a(t) \equiv b(t) \equiv c(t) \equiv 1$ and $h\left(x(t), x^{\prime}(t)\right)=a, g\left(x^{\prime}(t-r(t))\right)=b x^{\prime}(t-r)$ and $f(x(t-r(t)))=c x(t-r)$.

Remark 2.2 If $h\left(x(t), x^{\prime}(t)\right)=a$ and $a(t)=b(t)=c(t)=1$ in (1.1), the non-autonomous Equation (1.1) reduces to the autonomous equation considered in Sadek [4].

\section{The Boundedness of Solution}

Theorem 2 We assume that all the assumptions of Theorem 1 and

$$
\begin{aligned}
& \mid p(t, x, y, x(t-r(t)), y(t-r(t)), z \mid \leq q(t), \\
& \int_{0}^{t}|q(s)| \mathrm{d} s \leq P_{o}<\infty
\end{aligned}
$$

hold, where $P_{o}$ is a positive constant.

Then, there exists a finite positive constant $K$ such that the solution $x(t)$ of Equation (1.1) defined by the initial function

$$
x(t)=\phi(t), \quad x^{\prime}(t)=\phi^{\prime}(t), \quad x^{\prime \prime}(t)=\phi^{\prime \prime}(t)
$$

satisfies the inequalities

$$
|x(t)| \leq K, \quad\left|x^{\prime}(t)\right| \leq K, \quad\left|x^{\prime \prime}(t)\right| \leq K
$$

for all $t \geq t_{o} \geq 0$, where $\phi \in \mathcal{C}^{2}\left[t_{o}-r(t), t_{o}, \mathbb{R}\right]$ provided that

$$
\gamma<\min \left\{\frac{\left|2\left[\ell_{2}-\ell_{1}(b-\mu c)\right](1-\beta)\right|}{d_{o}\left(M_{2}+M_{1}\right)(1-\beta)+d_{o} M_{1}(\mu+1)} ; \frac{2\left[\mu a_{o}(1+a)-1\right](1-\beta)}{\mu d_{o}\left(M_{2}+M_{1}\right)(1-\beta)+d_{o} M_{2}(\mu+1)}\right\} .
$$

\section{Proof of Theorem 2}

As in Theorem 1, the proof of Theorem 2 depends on the scalar differentiable Lyapunov function $V\left(x_{t}, y_{t}, z_{t}\right)$ defined in (2.3).

Since $p \neq 0$, in (1.1).

In view of (2.16),

$$
\frac{\mathrm{d}}{\mathrm{d} t} V\left(x_{t}, y_{t}, z_{t}\right) \leq \dot{V}_{(1.2)}\left(x_{t}, y_{t}, z_{t}\right)+(y+\mu z)|p(t, x, y, x(t-r(t)), y(t-r(t)), z)|
$$


Since $\dot{V}_{(1.2)} \leq 0$ for all $t, x, y, z$ thus

$$
\frac{\mathrm{d}}{\mathrm{d} t} V\left(x_{t}, y_{t}, z_{t}\right) \leq-\delta_{4}\left(y^{2}+z^{2}\right)+(|y|+\mu|z|) q(t)
$$

Hence, it follows that

$$
\frac{\mathrm{d}}{\mathrm{d} t} V\left(x_{t}, y_{t}, z_{t}\right) \leq-\delta_{4}\left(y^{2}+z^{2}\right)+\delta_{5}(|y|+|z|) q(t) \leq \delta_{5}(|y|+|z|) q(t)
$$

for a constant $\delta_{5}>0$, where $\delta_{5}=\{1, \mu\}$.

Making use of the inequalities $|y|<1+y^{2}$ and $|z|<1+z^{2}$, it is clear that

$$
\frac{\mathrm{d}}{\mathrm{d} t} V\left(x_{t}, y_{t}, z_{t}\right) \leq \delta_{5}\left(2+y^{2}+z^{2}\right) q(t) \text {. }
$$

By (2.10), we have $\left(x^{2}+y^{2}+z^{2}\right) \leq D_{1}^{-1} V(x, y, z)$,

Hence,

$$
\frac{\mathrm{d}}{\mathrm{d} t} V\left(x_{t}, y_{t}, z_{t}\right) \leq \delta_{5}\left(2+D_{1}^{-1} V\left(x_{t}, y_{t}, z_{t}\right)\right) q(t)
$$

or

$$
\frac{\mathrm{d}}{\mathrm{d} t} V\left(x_{t}, y_{t}, z_{t}\right) \leq \delta_{6} q(t)+\delta_{6} V\left(x_{t}, y_{t}, z_{t}\right) q(t)
$$

where $\delta_{6}=\max \left\{2 \delta_{5}, \delta_{5} D_{1}^{-1}\right\}$.

Multiplying each side of this inequality by the integrating factor $\exp \left(-\delta_{6} \int_{0}^{t} q(s) \mathrm{d} s\right)$, we get

$$
\begin{aligned}
& \frac{\mathrm{d}}{\mathrm{d} t} V\left(x_{t}, y_{t}, z_{t}\right) \exp \left(-\delta_{6} \int_{0}^{t} q(s) \mathrm{d} s\right)-\delta_{6} V\left(x_{t}, y_{t}, z_{t}\right) q(t) \exp \left(-\delta_{6} \int_{0}^{t} q(s) \mathrm{d} s\right) \\
& \leq \delta_{6} q(t) \exp \left(-\delta_{6} \int_{0}^{t} q(s) \mathrm{d} s\right) .
\end{aligned}
$$

Integrating each side of this inequality from 0 to t, we get, where $V(0)=(x(0), y(0), z(0))$,

$$
V \exp \left(-\delta_{6} \int_{0}^{t} q(s) \mathrm{d} s\right)-V(0) \leq 1-\exp \left(-\delta_{6} \int_{0}^{t} q(s) \mathrm{d} s\right)
$$

or

$$
V\left(x_{t}, y_{t}, z_{t}\right) \leq V(0) \exp \left(\delta_{6} \int_{0}^{t} q(s) \mathrm{d} s\right)+\exp \left(\delta_{6} \int_{0}^{t} q(s) \mathrm{d} s\right)-1 .
$$

Since $\exp \left(-\delta_{6} \int_{0}^{t} q(s) \mathrm{d} s\right) \leq 1$ and using the fact that $\int_{0}^{t} q(s) \mathrm{d} s \leq P_{o}$ for all $\mathrm{t}$, this implies

$$
V\left(x_{t}, y_{t}, z_{t}\right) \leq V(0) \exp \left(\delta_{6} P_{o}\right)+\left[\exp \left(\delta_{6} P_{o}\right)-1\right] \text { for } t \geq 0 \text {. }
$$

Now, since the right-hand side is a constant, and since $V\left(x_{t}, y_{t}, z_{t}\right) \rightarrow \infty$ as $x^{2}+y^{2}+z^{2} \rightarrow \infty$, it follows that there exist a $K>0$ such that

$$
|x(t)| \leq K, \quad|y(t)| \leq K, \quad|z(t)| \leq K \quad \text { for } t \geq 0 .
$$

From the Equation (1.1) this implies

$$
|x(t)| \leq K, \quad\left|x^{\prime}(t)\right| \leq K, \quad\left|x^{\prime \prime}(t)\right| \leq K \quad \text { for } t \geq 0 .
$$

The proof of Theorem 2 is now complete.

Remark 3.1 If $r(t)$ is a constant, $g\left(x^{\prime}(t-r(t))\right)=g(\dot{x}), h\left(x(t), x^{\prime}(t)\right)=1$ and $p \neq 0$ in (1.1), the result obtained reduces to Omeike [6] and a result of Tunc [10].

\section{Conclusions}

The solutions of the third-order non-autonomous delay system are asymptotically stable and bounded according 
to the Lyapunov's theory if the inequalities (2.1) and (2.2) are satisfied.

Example 3.1 We consider non-autonomous third-order delay differential equation

$$
\begin{aligned}
& x^{\prime \prime \prime}(t)+\left(2+\frac{2}{|\sin t|+1}\right)\left[8+(x(t))+\left(x^{\prime}(t)\right)^{2}\right] x^{\prime \prime}(t) \\
& +\left(18+\frac{4}{|\sin t|+1}\right)\left[5 x^{\prime}(t-r(t))+\frac{x^{\prime}(t-r(t))}{\sin t+1}\right] \\
& +\left(9+\frac{4}{|\sin t|+1}\right)\left[12 x(t-r(t))+\frac{x(t-r(t))}{\sin t+3}\right] \\
& =\frac{1}{1+t^{2}+x^{2}(t)+x^{2}(t-r(t))+x^{\prime 2}(t)+x^{\prime 2}(t-r(t))+x^{\prime \prime}(t)}
\end{aligned}
$$

with equivalent system of (3.1) as:

$$
\begin{aligned}
x^{\prime}= & y \\
y^{\prime}= & z \\
z^{\prime}= & -\left(2+\frac{2}{|\sin t|+1}\right)\left[8+x^{2}(t)+y^{2}(t)\right] z(t) \\
& -\left(18+\frac{4}{|\sin t|+1}\right)\left[5 y+\frac{y}{\sin t+1}\right]-\left(9+\frac{4}{|\sin t|+1}\right)\left[12 x+\frac{x}{\sin t+3}\right] \\
& +\left(18+\frac{4}{|\sin t|+1}\right) \int_{t-r(t)}^{t}\left(5+\frac{1}{\sin (s)+1}\right) \mathrm{d} s+\left(9+\frac{4}{|\sin t|+1}\right) \int_{t-r(t)}^{t}\left(12+\frac{1}{\sin (s)+3}\right) \mathrm{d} s \\
& +\frac{1}{1+t^{2}+x^{2}(t)+x^{2}(t-r(t))+y^{2}(t)+y^{2}(t-r(t))+z(t)}
\end{aligned}
$$

comparing (1.2) with (3.2), it is easy to see that

$$
\begin{aligned}
& \left(2+\frac{2}{|\sin t|+1}\right)=a(t) \geq a_{o}=3 \\
& \left(18+\frac{4}{|\sin t|+1}\right)=b(t) \geq b_{o}=20 \\
& \left(9+\frac{4}{|\sin t|+1}\right)=c(t) \geq c_{o}=11
\end{aligned}
$$

The function $f(x)=\left[12 x+\frac{x}{\sin t+3}\right]$, it is clear from the equation that

$$
\frac{f(x)}{x} \geq 12=\delta_{1}>0, \quad x \neq 0
$$

The function $g(y)=\left[5 y+\frac{y}{\sin t+1}\right]$, it is clear from the equation that

$$
\begin{aligned}
& \frac{g(y)}{y} \geq 5=b>0, \quad y \neq 0 \\
& f^{\prime}(x) \leq 12=c, \quad c>0
\end{aligned}
$$




$$
\begin{aligned}
& h(x, y)=8+x^{2}+y^{2} \\
& 8+x^{2}+y^{2} \geq 8=1+7, a=7 \\
& \frac{5}{12}>\mu>\frac{1}{3}, \text { we choose, } \mu=\frac{7}{20} \\
& d_{o} \geq 20 \geq 11 \geq \ell_{1}, \text { we choose, } d_{o}=25 \text { and } \ell_{1}=10
\end{aligned}
$$

also,

$$
\begin{aligned}
& 1 \leq \ell_{2} \leq 8, \text { we choose, } \ell_{2}=4 \\
& \left|f^{\prime}(x)\right|=12=M_{1} \\
& \left|g^{\prime}(x)\right|=5=M_{2}
\end{aligned}
$$

Since

$$
0<\beta<1 \text {, we choose, } \beta=\frac{1}{2} \text {, }
$$

we have

$$
\gamma<\min \left\{\frac{16}{3705}, \frac{84}{1459}\right\}=\frac{16}{3705}
$$

It follows that $|r(t)| \leq \frac{16}{3705}$, if the delay is increased beyond this range a limit cycle appear, followed eventually by a period-doubling cascade leading to chaos.

Finally,

$$
\begin{aligned}
& p(t, x(t), y(t), x(t-r(t)), y(t-r(t)), z(t)) \\
& =\frac{1}{1+t^{2}+x^{2}(t)+x^{2}(t-r(t))+y^{2}(t)+y^{2}(t-r(t))+z(t)} \leq \frac{1}{1+t^{2}}
\end{aligned}
$$

and

$$
\int_{0}^{\infty} q(s) \mathrm{d} s=\int_{0}^{\infty} \frac{1}{1+s^{2}} \mathrm{~d} s=\frac{\Pi}{2}<\infty .
$$

Thus, all assumptions of Theorem 1 and Theorem 2 are held. That is, zero solution of Equation (1.1) is asymptotically stable and all the solutions of the same equation are bounded.

\section{References}

[1] Afuwape, A.U., Omari, P. and Zanalin, F. (1989) Nonlinear Pertubations of Differential Operators with Nontrivial Kernel and Applications to Third-Order Periodic Boundary Problems. Journal of Mathematical Analysis and Applications, 143, 35-36. http://dx.doi.org/10.1016/0022-247X(89)90027-9

[2] Cronin, J. (1997) Some Mathematics of Biological Oscillations. SIAM Review, 19, 100-137. http://dx.doi.org/10.1137/1019007

[3] Rauch, L.L. (1950) Oscillations of a Third-Order Nonlinear Autonomous System in Contributions to the Theory of Nonlinear Oscillations. Annals of Mathematics Studies, 20, 39-88.

[4] Sadek, A.I. (2003) On the Stability and Boundedness of a Kind of Third Order Delay Differential System. Applied Mathematics Letters, 16, 657-662. http://dx.doi.org/10.1016/S0893-9659(03)00063-6

[5] Sadek, A.I. (2005) On the Stability of Solutions of Some Non-Autonomous Delay Differntial Equations of Third Order. Asymptotic Analysis, 43, 1-7.

[6] Zhu, Y.F. (1992) On Stability, Boundedness and Existence of Periodic Solution of a Kind of Third Order Nonlinear Delay Differential System. Annals of Differential Equations, 8, 249-259.

[7] Afuwape, A.U. and Omeike, M.O. (2008) On the Stability and Boundedness of Solutions of a Kind of Third Order De- 
lay Differential Equations. Applied Mathematics and Computation, 200, 444-451. http://dx.doi.org/10.1016/j.amc.2007.11.037

[8] Ademola, A.T. and Aramowo, A.T. (2013) Uniform Stability and Boundedness of Solutions of Non-Linear Delay Differential Equations of Third Order. Mathematical Journal of Okayama University, 55, 157-166.

[9] Yao, H. and Meng, W. (2008) On the Stability of Solutions of Certain Non-Linear Third Order Delay Differential Equations. International Journal of Non-Linear Science, 6, 230-237.

[10] Ademola, A.T., Ogundare, B.S., Ogundiran, M.O. and Adesina, O.A. (2015) Stability, Boundedness and Existence of Periodic Solutions to Certain Third-Order Delay Diffrential Equation with Multiple Deviating Arguments. International Journal of Differential Equations, 2015, Article ID: 213935.

[11] Omeike, M.O. (2009) Stability and Boundedness of Solutions of Some Non-Autonomous Delay Differential Equation of the Third Order. Analele Stiintifice Ale Universitattii “Alexandru Ioan Cuza” Din Iasi Matematica, 55, 49-58.

[12] Tunc, C. (2009) Boundedness in Third Order Nonlinear Differential Equations with Bounded Delay. Boletin de Mathematicas, 16, 1-10.

[13] Tunc, C. (2006) New Results about Stability and Boundedness of Solutions of Certain Non-Linear Third Order Delay Differential Equations. The Arabian Journal for Science and Engineering, 31, 185-196. 